\title{
A Newly Developed Stereotactic Robot with Detachable Drive for Neurosurgery
}

\author{
Ken MASAMUNE, L.H. JI, Makoto SUZUKI, Takeyoshi DOHI, *Hiroshi ISEKI, \\ *Kintomo TAKAKURA
}

\author{
Graduate school \& Faculty of Engineering, the University of Tokyo \\ *Dept. of Neurosurgery, Tokyo Women's Medical College \\ e-mail: masa@miki.pe.u-tokyo.ac.jp
}

\begin{abstract}
This paper describes the development of a needle insertion manipulator for stereotactic neurosurgery. This robot fulfils the requirements of having both a safe mechanical design and the capacity for being sterilized. Many kinds of robots are examined in neurosurgery. Their purpose is the precise positioning of surgical instruments such as biopsy needles, electrodes etc. Some are already available commercially and have been proven useful in the operating theatre. However, their clinical application is limited by specific problems including cost, safety, positioning requirements, maintenance requirements. The main problems have been with the safety of the mechanical design and difficulties with sterilization and disinfecting pre- and post operatively. The manipulator described in this report achieves mechanical safety and has the capacity for cover-sheet-free sterilization. The manipulator has three major components: the main mechanical component (with 6 degrees of freedom), the torque transmission component, and the electric motor, which cannot be sterilized. The electrical parts are detachable. Using this mechanism, we can clearly separate the surgical area from the mechatronics components. In this paper, the basic design and the prototype development and testing are described.
\end{abstract}

\section{Introduction}

Since the end of 1980s, many kinds of robotic technologies have been applied to stereotactic surgery, especially neurosurgery [1][2][3][6][7]. MINERVA in Switzerland, and NEUROMATE (IMMI) in France are now currently considered representative of advanced surgical robots for neurosurgery. The main purpose of these robots is the precise positioning of surgical instruments such as bionsy needles, electrodes and X-ray nesdles [4]. However, even those which are commercially available are limited in their clinical application by the following problems: cost, safety, maintenance requirements and the robot's mass and weight. As safety is the most important concern, our priority was to design a robot that was mechanically safe. The redundant working area of the robot is dangerous not only for the patient but also 
for the surgeons, even when safety has been built into the software and mechanical movement is slow. The next concern is to address the sterilization and disinfecting problem. It is common practice for advanced medical robots to be covered with sterilized plastic sheets. These are necessary when the robot is used in clinical settings. The reason for the cover sheet is that robots have many electrical parts, which cannot be sterilized because of problems arising during sterilization. Rusting, breakage and malfunction of the robot can result from autoclaving or ethylene oxide gas (EOG) sterilization.

In this paper, the requirements for a medical manipulator and a newly designed needle insertion medical robot for stereotactic neurosurgery are described, with a special focus on solving the sterilization problem.

\section{System requirements}

Stereotactic neurosurgery requires precise positioning of the insertion instruments, which perform biopsy, inject drugs, or position electrodes. Precision is the main concern, because it is crucial that any functional areas and major blood vessels of the brain be avoided. The acceptable positioning tolerance for stereotactic surgery is about $1-3 \mathrm{~mm}$, including distortions due to the imaging system. Stereotactic neurosurgery is usually performed with a frame, which is connected to the bed. The frame builds the absolute reference system to determine the position of insertion of the neurosurgical tools. Before conducting surgery, X-ray, CT and MRI scans are used to define the exact anatomical location of the target, relative to the stereotactic frame.

Considering these requirements, it appeared that the most direct approach was to redesign an industrial robot to position the instruments. Industrial robots are very good at controlling localization, maintaining a constant position, etc. However, significant changes are needed to satisfy the requirements of the operating theatre. Neither the hardware nor the software of industrial robots is designed for surgery and cannot be applied in a medical setting. Therefore, we propose a completely new design, based upon specific clinical demands. Medical robots have different requirements to industrial robots. We listed most important item below.

Safety: Prevention of accidents must be given the highest priority. In particular, safety has to be built into the mechanical design. There should be no possibility of interference with the patient except for the required movements. If there is a power failure when a medical robot is being used, the surgeon must be able to continue the operation manually. In comparison, if there is a power failure in an industrial setting, one can simply wait for the power to return.

Sterilization, Washable structure: The parts which are in direct contact with the patient have to be completely sterile and therefore must be steralizable. Parts without direct human contact can be covered with sterile sheets. However, we prefer to use fewer sheets as the manipulator often tears the sheets, thus breaking the sterile area. After the operation, the manipulator should be disinfected. Therefore the shape and the structure of its mechanism must be simple and easy to wash. 
No trial movement permitted: The main purpose of today's surgical robot is basically to remove human tissue (for biopsy, hip prosthesis placement, etc.). Therefore, it is clear that test runs are not permitted.

In addition, we need different functions for each medical procedure. User friendly interfaces are also required.

While we consider the safety of medical robots to be the most important issue, the next major issue is that of sterilization. While most neurosurgical robots have significant safety measures, there is a need for a lot more discussion of the sterilization problem. As long as sterilized plastic cover sheets are used, the robot will remain a 'special' instrument in clinical settings. In the next section we describe the design of our new manipulator, which fulfills the above requirements as a medical robot.

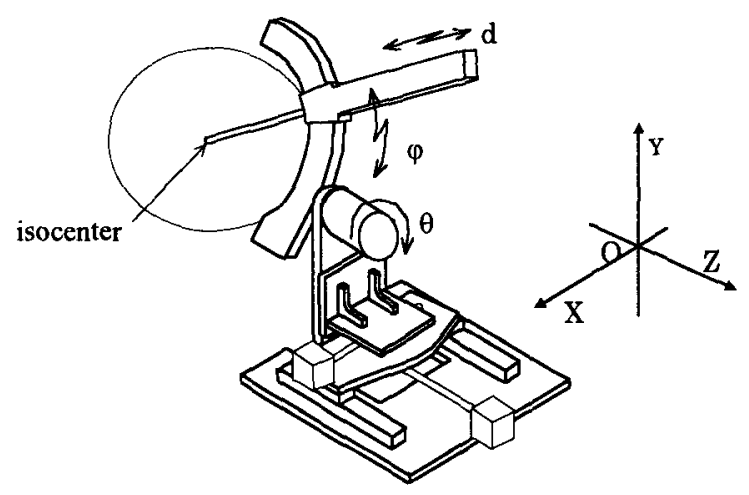

Fig. 1. Principle mechanism of an isocentric needle insertion manipulator

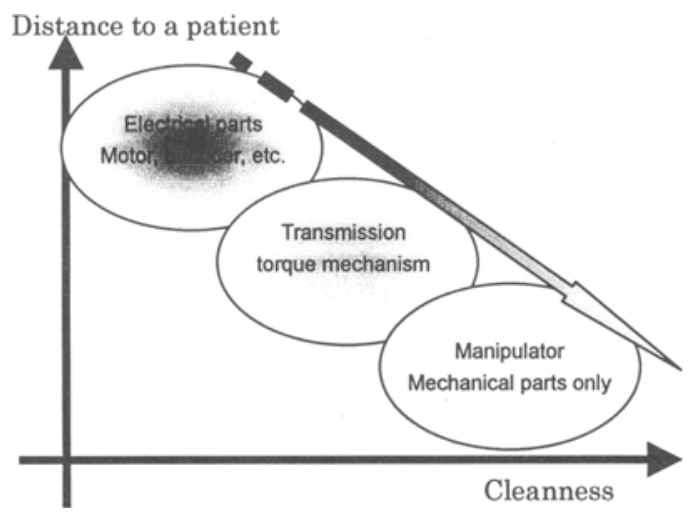

Fig. 2. Level of sterilization of the manipulator 


\section{A new mechanism}

The basic mechanical structure of our robot is the same as our former prototype [3]. The concept behind our manipulator is a motorized stereotactic frame with six degrees of freedom, as shown in Fig. 1. This isocentric structure has the advantage of canceling the positional error in the center point, which is the target. By this structure, the robot avoids collision with the patient's head except for the insertion axis $d$. Compared to redundant degree of freedom robots, this mechanism is a safety feature built into the robot's design. Having an identical geometrical structure to a conventional stereotactic frame enables the surgeon to operate the robot manually in emergency situations. Furthermore, the reliability of the control is high because all axes are independent of each other. The almost same mechanism are applied to use in other works such as [7], however, our system is designed so that it can be positioned on the CT imaging scanner bed, so the size of the manipulator is restricted. During the operation, the procedure can be watched on transverse CT images without any artifact. CT images are also directly used to acquire registration between the patient coordinate and the manipulator's coordinate.

Another particular feature of this robot is its torque transmission mechanism, which solves the sterilization problem. Autoclave and EOG, the typical sterilization methods, require components to endure high pressure and temperature or acid liquid. Many electrical components are not able to withstand these conditions so only mechanical components can be sterilized.

To overcome this problem, we proposed a basic design whereby we separate mechanical components from electrical components. Fig. 2 shows this design concept. This separation means that we can safely bring the mechanical components closer to the surgical area. The distance between the patient and the manipulator is decreased and the risk of the manipulator contaminating the surgical field is also decreased.

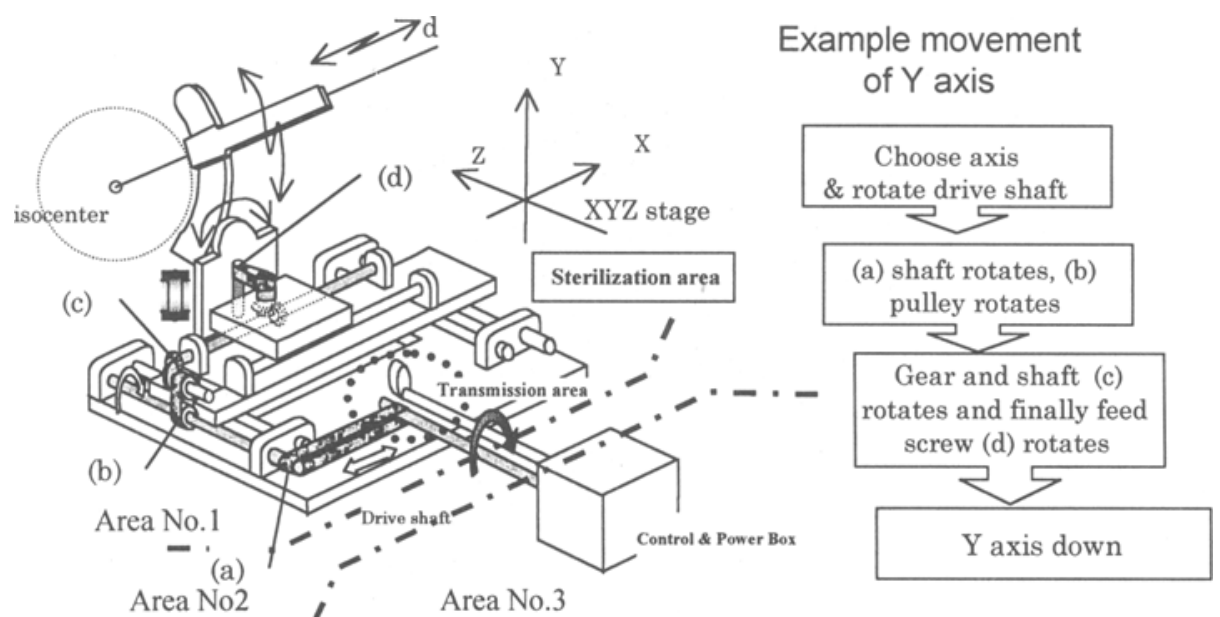

Fig. 3. Mechanism of the needle insertion manipulator and principle of the movement of $Y$ axis 
With this design, our new robot can be separated into three parts, two mechanical areas which can be sterilized, and one mechanical and electrical area which cannot be sterilized. Fig. 3 shows a mechanical outline of the manipulator. Area No.1 is the main mechanism. It has 6 degrees of freedom and consists only of mechanical parts. Area No. 2 is also assembled with sterilizable mechanical parts, the role of which is to transfer rotating power from the power source Area No.3 to the mechanical area No.1. Area No.3, the power source, includes three pulse motors which cannot be sterilized. In the right side of Fig.3, one example of the moving $\mathrm{Y}$ axis is described. The rotating power of each axis is transferred via Area No.2 to the gear transmission system in Area No.1.

We used aluminum for the main parts of the manipulator. The drive shafts and transmission shaft are made of stainless steel (SUS304) to transfer the power. These are all sterilizable materials and are autoclave tolerant. In addition, we can easily separate the XYZ axis anc the arc axis for sterilization.

Feed screws are used for the XYZ axis and the insertion axis d. Table 1 shows the specifications of the manipulator. Fig. 4 is a photograph of the first prototype robot.

Table 1. Specification of the manipulator

\begin{tabular}{|c|c|}
\hline Mechanism (DOF) & $\mathrm{XYZ}$ axis(3), rotation axis(2), insertion axis(1) \\
\hline $\begin{array}{l}\text { Actuator \& Feed } \\
\text { mechanism }\end{array}$ & $\begin{array}{l}\text { 3 pulse motors } \\
\text { XYZ, rotation axis: gear, spline shaft, feed screw for transmission } \\
\text { Insertion axis : flexible shaft \& feed screw or manual }\end{array}$ \\
\hline \multicolumn{2}{|l|}{ Moving range } \\
\hline Depth & $0-110 \mathrm{~mm}$ \\
\hline Rotate axis & $-45-45$ (deg.) \\
\hline Latitude axis & $10-70$ (deg.) \\
\hline $\mathrm{XYZ}$ stage & $0-90(\mathrm{~mm})$ \\
\hline Weight & $7(\mathrm{~kg})$ (including motors) \\
\hline Size & $320(w) \times 360(d) \times 440(h) \mathrm{mm}^{3}$ \\
\hline
\end{tabular}

\section{Experimental Result}

We checked the movement of each axis and confirmed that the XYZ stage and the $\mathrm{d}$ axis moved correctly. The accuracy of the movement was within $1 \mathrm{~mm}$ for each axis. Of course, by using gears, backlashes were over $5 \mathrm{~mm}$, which is not small. The reason for this is the many gears and rubber belts used in the transmission mechanism. However, we plan to use a "one-way movement" and thus backlash will no longer be a problem. In addition, by using CT images, we can check the exact position of the manipulator before the insertion task.

With the arc axis, we could not always confirm its precise and correct movement. Sometimes the pulse motor stopped even though the control signal was sent correctly. This was because of an overload, caused by a manufacturing error, and friction. 
Currently we are working on improvements and are manufacturing a new manipulator. For sterilization testing we sterilized each part a minimum of 3 times by autoclaving, and no damage (distortion, rusting etc.) was found. Fig. 5 shows the manipulator installed into the X-ray CT imaging system. It is put on the scanner's bed and its size is small enough to be set in the gantry.

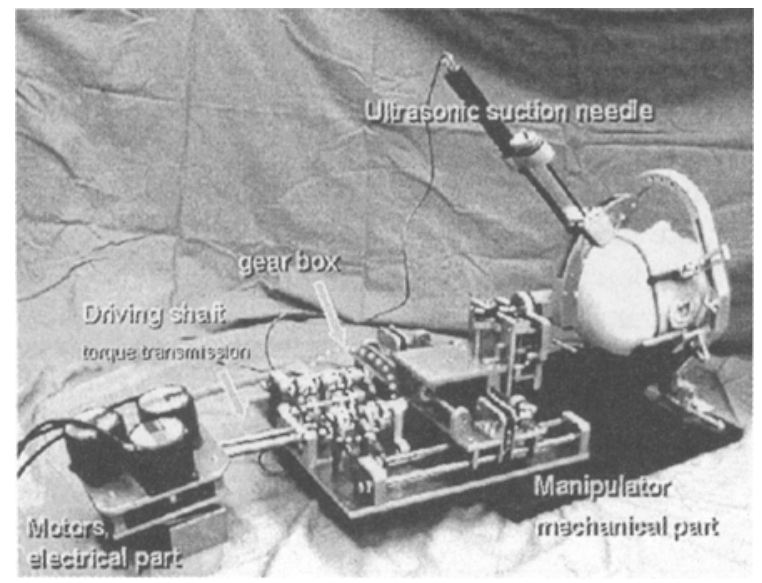

Fig. 4. Overview of the manipulator

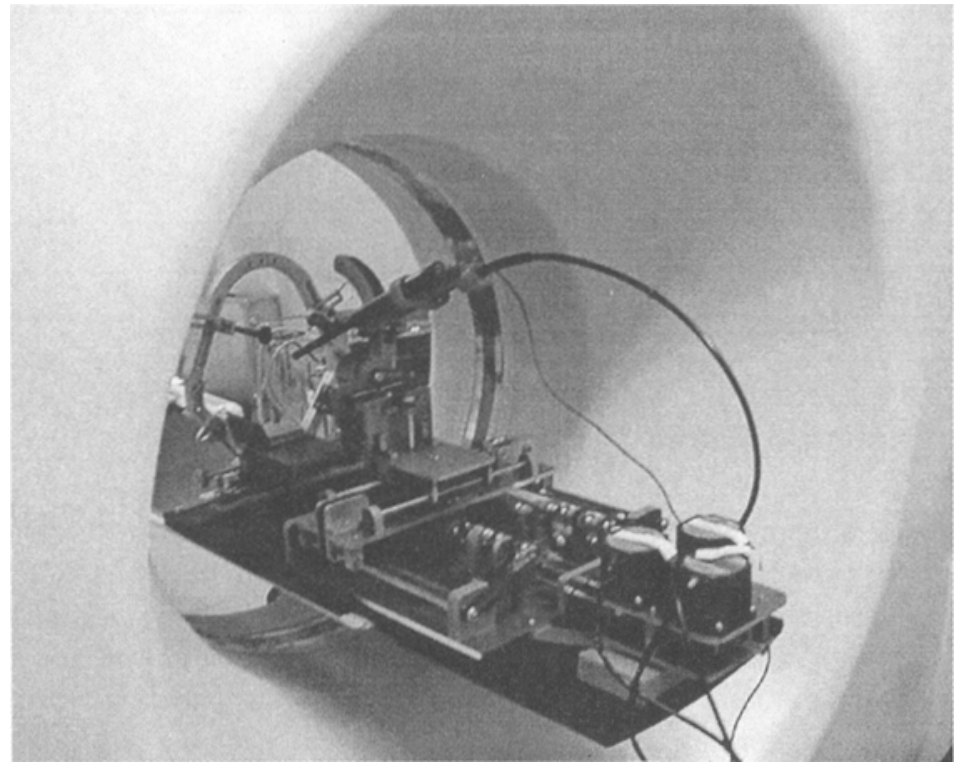

Fig. 5. Manipulator installed in the $\mathrm{X}$-ray imaging system 


\section{Discussion}

\section{Clinically oriented design}

Mechanical safety was achieved by using the virtual sphere principle. The size of our manipulator is small enough for it to be settled on the X-ray CT scanner's bed, which increases the precision of positioning. At this operation, this manipulator only does positioning and needle insertion, but not more complex procedures. For safety, redundant movement is not necessary.

Sterilization is the next important problem in the development of medical robotics. In this paper, we suggest our concept of separating mechanical components from detachable electrical components using a transmission shaft. Only the electrical components that are some distance away from the surgical area require covering with drapes. Many medical rcbots, like the stereoscopic microscope, use plastic cover sheets. However, we consider it is not appropriate to merely use plastic cover sheets for medical robots. This manipulator is only inserting the needle to the target. It seems more complicated task to use such a sterilized cover sheet.

Cost should be also carefully thought about. Much expensive robots are not acceptable to use for such a simple task. Considering the total number of applicable operations and patients in one hospital in one year, only over 100 to 200 operations would require the medical robot. So, it makes no sense to make a large investment in such manipulators for this rate of use. So a low cost is preferable. Because of simple structure, our manipulator is not as expensive as other medical robots when controlling units etc. are included.

\section{Future work}

Our immediate priority is to improve the manipulator fabrication. After further experimentation and testing, we are expecting to use it in a clinical setting.

The next stage will probably to develop MRI compatible type using mechanism written above. We succeeded to develop an MRI compatible manipulator for neurosurgery in 1995 [5]. Because of the strong magnetic field in the MRI scanner, we used ultrasonic motors and encoders made of non-ferromagnetic materials to ensure that there was few artifact in the scanned image. However, this manipulator also had a sterilization problem in clinical settings. With these non-ferromagnetic electrical materials, it would be possible to develop an MRI compatible manipulator, because all the materials used already are non-ferromagnetic, except for the motors and a few bearing parts inside the gear transmission shaft.

We have also developed a surgical simulation of needle puncturing [3]. To use this simulation clinically, the registration problem needs to be considered. Registration is required not only between the manipulator and the patient's head, but also between the manipulator and the $\mathrm{X}$-ray image. 


\section{Conclusion}

Mechanical safety is achieved by using the virtual sphere principle. We separated the mechanical components from the electrical components to solve the sterilization problem. We conclude that this prototype is first in demonstrating new principles of how medical robots can be developed, taking full account of safety and sterilization considerations. This research is partly supported by JSPS-RFTF96P00801 program.

\section{References}

[1] D. Glauser, H. Fankhauser, M. Epitaux, J.-L. Hefti, A. Jaccottet: Neurosurgical Robot Minerva: First Results and Current Developments, J. of Image Guided Surgery, 1:266272,1995 .

[2] F. Badano, et. al., The NEURO-SKILL Robot: a New Approach for Surgical Robot Development, Proc. MRCAS95, pp.318-323, 1995.

[3] K. Masamune, et al., Robots for neurosurgery, Advanced Robotics, Vol.10, No.4, pp.391$401,1996$.

[4] K. Masamune, et al., Development of a CT-guided Neurosurgical Manipulator for Photon Radiosurgery System, CAR97, p.1035, 1997.

[5] K. Masamune, et al., Development of a MRI Compatible Manipulator for Stereotactic Neurosurgery, Image Guided Surgery, 1995.

[6] Yamauchi Y., Ohta Y., Dohi T., Kawamura H., Tanikawa T., Iseki H.: A needle insertion Manipulator for X-ray CT image guided neurosurgery, J.of LST, vol. 5-4, 814-821, 1993.

[7] Kelly PJ, Goerss SJ, Kall BA: Evolution of contemporary instrumentation for computerassisted stereotactic surgery, Surgical Neurology 30(3):204-15, 1988 